\title{
Sedative effects and risk factors for desaturation during transport after general anesthesia with sevoflurane in pediatric ambulatory inguinal herniorrhaphy cases: an observational study
}

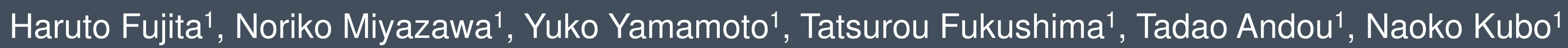 \\ 1) Tokyo Metropolitan Children's Medical Center
}

\section{Introduction}

Desaturation is one of the major potential complications during the transport of postoperative patients from the operating room to the post-anesthesia care unit (PACU). However, children are at high risk not only for desaturation but also for emergence agitation especially after general anesthesia using sevoflurane (1). Some favor transporting children under sedation in order to prevent emergence agitation.

Most current reports demonstrated no difference in the incidence of pediatric respiratory complications resulting from laryngeal mask airway (LMA) removal whether this was done under deep anesthesia or when the patient was awake (2)(3). However, we were unable to find reports on the quality of sedation during transport to the recovery unit after LMA removal under deep anesthesia with sevoflurane.

The goal of this study was to assess the efficacy and safety of transport immediately after deep sevoflurane anesthesia.

\section{Material and Methods}

A retrospective, single center study conducted at Tokyo Metropolitan Children's Medical Center.

$>325$ cases of elective ambulatory inguinal herniorrhaphy from 6 months to 15 years of age in a 1-year period from July 2015 to June 2016

Examination by an anesthesiologist in the one month prior to surgery

\begin{tabular}{|l|l|}
\hline & The ambulatory surgery criteria \\
$\bullet$ & ASA-PS 1 or 2 \\
$\cdot$ & No severe systemic diseases \\
$\bullet$ & No asthma attack \\
$\cdot$ & No convulsion within one year \\
\hline
\end{tabular}

Examination by the anesthesiologist in charge on the day of surgery The operation was postponed due to obvious upper respiratory symptoms

Anesthesia

- No premedication

Induction with sevoflurane, nitrous oxide, and oxygen

Intravenous catheter insertion

$0.01-0.02 \mathrm{mg} / \mathrm{kg}$ atropine sulfate

LMA insertion

Ultrasound-assisted ilioinguinal-iliohypogastric nerve block

Maintenance by $3 \%$ sevoflurane with air and oxygen

Herniorrhaphy

LMA removal under 3\% sevoflurane

Promptly transferred to the PACU with $3 \mathrm{~L} /$ min oxygen-mask

1

Observation until discharge $\quad *$ A protocol exists but is not mandatory

\section{$>$ Definition}

- Hypoxemia was defined as $\mathrm{SpO} 2<90 \%$.

- Sedation was defined as a state in which the patient remains immobile with closed eyes until touched, or a state of wakefulness in which the patient does not move.

$>$ Statistical analysis

- The primary outcome: the incidence of agitation and hypoxemia during transport

- The secondary outcome: the risk factors predicting hypoxemia, comparing the incidence of hypoxemia with age, preoperative snoring, upper airway tract symptoms (cough, fever, rhinorrhea, nasal congestion, pharyngeal pain), anesthetic time, and end-tidal sevoflurane concentration at LMA removal (R software version 3.0.0, Logistic regression analysis, $\mathrm{p}<0.05)$.

\section{Results}

Table 1. Descriptive analysis of recorded variables

\begin{tabular}{ll|}
\hline Variables & $n=323$ \\
\hline Age, months & $52.7 \pm 30.9$ \\
Sex (M/F) & $183(56.7) / 140(43.3)$ \\
Weight (kg) & $16.5 \pm 6.7$ \\
Height $(\mathrm{cm})$ & $100.6 \pm 18.9$ \\
Laterality (unilateral/bilateral) & $306(94.7) / 17(5.3)$ \\
Preoperative snoring (yes/no) & $22(6.8) / 301(93.2)$ \\
Score for upper airway symptoms (2 or more than 2/<2) & $47(14.6) / 276(85.4)$ \\
Duration of anesthesia (min) & $46.6 \pm 10.8$ \\
Duration of operation (min) & $15.5 \pm 8.0$ \\
Use of intraoperative additional analgesia (yes/no) & $39(12.1) / 284(87.9)$ \\
End-tidal sevoflurane concentration (\%) & $2.79 \pm 0.57$ \\
Duration of transport (min) & $5.9 \pm 2.9$ \\
Duration of postoperative observation (min) & $188.1 \pm 40.2$ \\
Postoperative vomiting (yes/no) & $6(1.9) / 317(98.1)$ \\
Use of postoperative analgesia (yes/no) & $87(26.9) / 236(73.1)$ \\
\hline Results are expressed as the mean \pm SD for quantitative data and as number and percentage for qualitative data
\end{tabular}

Results are expressed as the mean + SD for quantitative data and as number and percentage for qualitative data

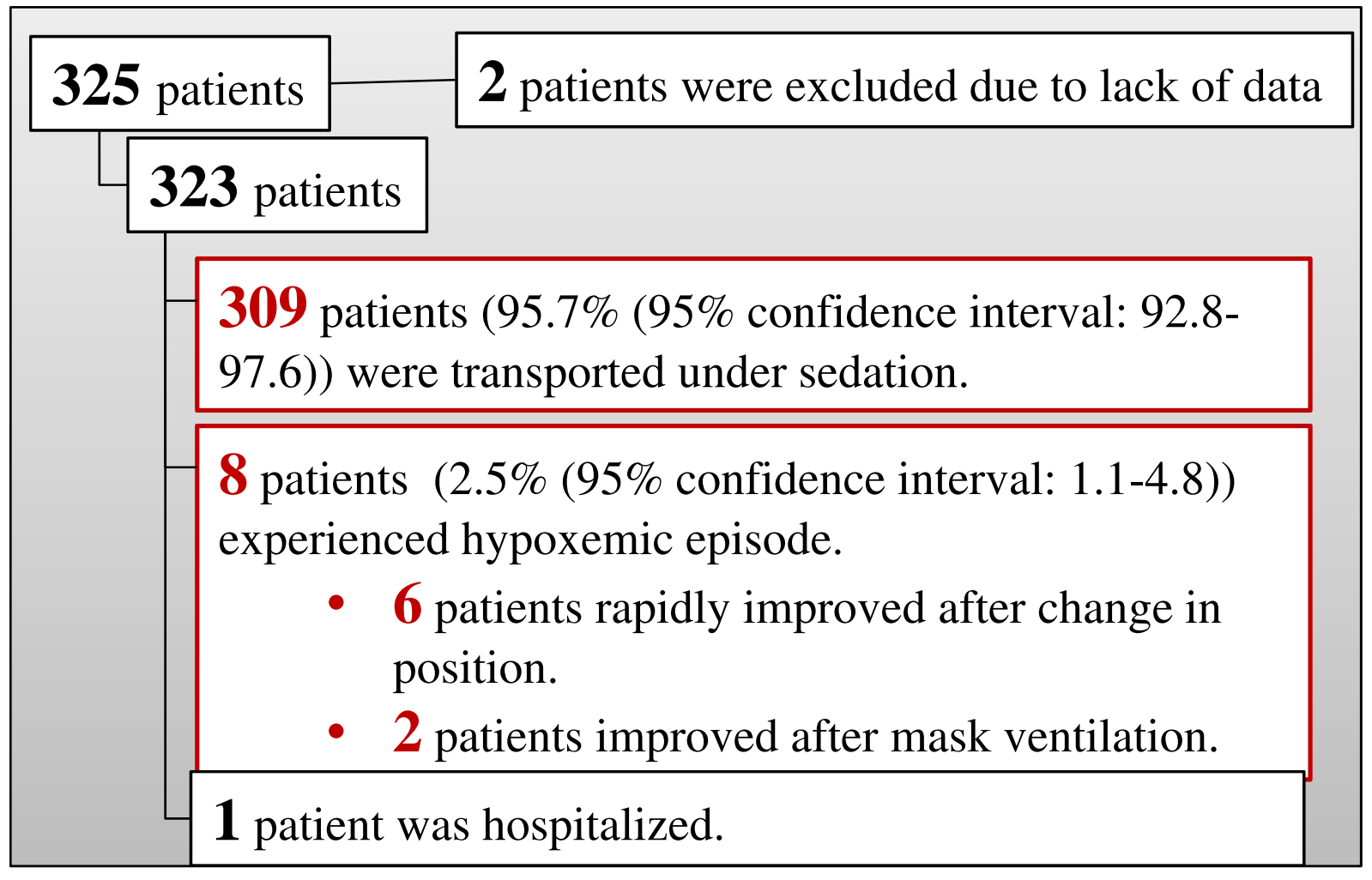

Table 2. Univariate analysis

\begin{tabular}{lllll}
\hline & \multicolumn{4}{c}{$95 \%$ confidence interval } \\
Variable & Odds ratio & Lower & Upper & $\mathrm{P}$ \\
\hline Age in month & 1.0180 & 0.9983 & 1.0380 & 0.0733 \\
Sex (male as dummy variable) & 1.2827 & 0.3013 & 5.4610 & 0.7360 \\
Preoperative snore & 4.9165 & 0.9320 & 25.9367 & 0.0605 \\
\hline Respiratory tract infection score $>1$ & 1.9999 & 0.3914 & 10.2186 & 0.4050 \\
Duration of anesthesia & 1.0197 & 0.9659 & 1.0766 & 0.4807 \\
End-tidal sevoflurane concentration & 0.8775 & 0.2800 & 7.9267 & 0.8226 \\
\hline
\end{tabular}

Table 3. Multivariate analysis

\begin{tabular}{lllllll}
\hline Table 3. Multivariate analysis & & & \multicolumn{3}{c}{$95 \%$ confidence interval } \\
Variable & Coefficient & Standard error & $\mathrm{P}$ & Odds ratio & Lower & Upper \\
\hline (Intercept) & -9.3687 & & & & & \\
Age in month & 0.0289 & 0.0129 & $0.0255{ }^{*}$ & 1.0293 & 1.0035 & 1.0557 \\
Sex (male as dummy variable) & 0.2408 & 0.7943 & 0.7618 & 1.2722 & 0.2682 & 6.0345 \\
\hline Preoperative snore & 1.8400 & 0.9112 & $0.0435{ }^{*}$ & 6.2963 & 1.0554 & 37.5619 \\
\hline Respiratory tract infection score $>1$ & 0.8369 & 0.8674 & 0.3346 & 2.3093 & 0.4218 & 12.6422 \\
Duration of anesthesia & 0.0316 & 0.0324 & 0.3302 & 1.0321 & 0.9685 & 1.0998 \\
End-tidal sevoflurane concentration & 0.6542 & 0.7225 & 0.3652 & 1.9236 & 0.4668 & 7.9267 \\
\hline
\end{tabular}

References

1) Costi D, et al. Effects of sevoflurane versus other general anesthesia on emergence agitation in children Cochrane Database of Systematic Reviews 2014, Issue 9. Art. No.: CD007084.

2) Jeong-Soo Park, et al. A randomized controlled trial comparing Laryngeal Mask Airway removal during adequate anesthesia and after awakening in children aged 2 to 6 years. Journal of Clinical Anesthesia 2012; 24: $537-541$.

3) J. Lee, et al. Removal of the laryngeal tube in children: anaesthetized compared with awake. British Journal of Anaesthesia 2007; 98(6): 802-5.

\section{Conclusion}

Preventing agitation during transport after anesthesia with sevoflurane in children is challenging. Residual sevoflurane provided adequate sedation but $2.5 \%$ of patients experienced hypoxemia. Preoperative snoring was related to the incidence of hypoxemia. The presence of well-trained staff during transport is strongly advised. In the future, more effective, safer methods of transporting children postoperatively should be considered. 